\section{Arbeit und Innovation im demografischen Wandel}

\section{Demografischer Wandel}

Megatrend Demografie: weniger, älter, bunter

Kein Zweifel, der „Megatrend Demografie“ ist in der öffentlichen Diskussion angekommen. Was vor zehn Jahren noch als Spezialfeld von Forschern und Bevölkerungsstatistikern galt, ist heute aus den Medien, der öffentlichen Diskussion und der Strategieentwicklung informierter Akteure nicht mehr wegzudenken. Zu den Fakten des demografischen Wandels sind bereits an vielen Stellen einschlägige Daten und Trends publiziert worden, die hier nicht wiederholt werden sollen Die unmittelbaren Folgen des demografischen Wandels stellen sich wie folgt dar:

- Die Bevölkerung wird weniger, weil sie sich nicht mehr ausreichend reproduziert.

- Die Menschen werden älter, weil es weniger junge Menschen gibt und gleichzeitig die Lebenserwartung steigt.

- Die Bevölkerung wird in mehrfacher Hinsicht „bunter" werden durch Binnenwanderung, Einwanderung und eine stärkere Erwerbsbeteiligung bestimmter Gruppen am Arbeitsmarkt.

Festzustellen ist aber auch, dass, trotz dieses Konsenses, die Komplexität des Phänomens seine planerische Antizipation und praktische Bewältigung in erheblichem Maße erschwert. Dazu tragen eine Reihe von Faktoren bei:

- Je weiter man in der Betrachtungsebene hinabsteigt, umso unterschiedlicher sind die jeweiligen Rahmenbedingungen und umso schwieriger sind mögliche Auswirkungen zu prognostizieren. Je höher die Auflösung der Betrachtung, umso mehr andere Wirkungsfaktoren treten hinzu. Die Bevölkerungsentwicklung der EU und von Gesamtdeutschland lässt sich einigermaßen genau bestimmen. Auf der Ebene eines Kreises oder gar eines einzelnen Unternehmens kann die Ausgangslage anders sein, treten erhebliche Schwankungen auf und sind zahlreiche weitere Faktoren zu berücksichtigen ${ }^{1}$.

- Der Leidensdruck von Betroffenen ist unterschiedlich ausgeprägt. Je nach dem, ob in einer Region Bevölkerungszuwachs oder -rückgang stattfindet, ob eine Branche bereits heute Fachkräftemangel aufweist oder nicht, ob man für sich persönlich annimmt, mit 58 Jahren in den Ruhestand gehen zu können oder bis 68 arbeiten zu müssen, ergeben sich unterschiedliche Bewertungen und Handlungsbereitschaften.

- Verschiedene Akteure haben unter Umständen unterschiedliche Interessen. Bundesländer, Kreise und Städte, Unternehmen und Beschäftigte, Junge, Mittelalte und Alte, Produzenten und Dienstleister, usw. haben immer auch eigene Prioritäten beim Blick auf demografische Veränderungen und deren Folgen. Es muss nicht zwingend angenommen werden, dass ihre Interessen immer konfliktbehaftet sind im Sinne eines „Nullsummenspieles“. Anderseits wäre es ebenfalls verfehlt, einen immer bereits vorhandenen geeinten Willen zum Handeln vorauszusetzen.

- Schließlich müssen wir uns ebenfalls stets vor Augen halten, dass wir es mit einem für uns einzigartigen Geschehen zu tun haben. Daher liegen wenig Erfahrungen und fundierte Erkenntnisse vor, die wir zur Entwicklung von Bewältigungsstrategien und zur Verbreitung von guter Praxis nutzen können. Wenn Erfahrungen vorliegen, sind zum Teil erhebliche Unterschiede in den historischen, gesellschaftlichen, politischen, geografischen und wirtschaftlichen Rahmenbedingungen in Ansatz zu bringen.

\section{Auswirkungen auf das gesellschaftliche Teilsystem „Wirtschaft“}

Vor diesem Hintergrund und in dem Bewusstsein, dass die Wettbewerbsfähigkeit der Unternehmen und der Erhalt von Arbeitsplätzen ein gemeinsames Anliegen sind, haben sich in Nordrhein-Westfalen die Landesvereinigung der Arbeitgeberverbände, der Deutsche Gewerkschaftsbund und das damalige Ministerium für Wirtschaft und Arbeit zu einer gemeinsamen Initiative „Arbeit und Innovation im demografischen Wandel“" $\left(\operatorname{arbid}^{2}\right)$ zusammengefunden.

Wir wollen zunächst deutlich machen, um welche Betrachtungsebene es uns geht. Gesamtbetrachtungen der volkswirtschaftlichen Wirkungen demografischer Entwicklungen und ihrer Konsequenzen sind nicht Gegenstand unserer Arbeit. Uns geht es um die betriebliche und betriebswirtschaftliche Ebene der Unternehmen in NRW. Dazu sollen zunächst die wesentlichen Wirkungsstränge des demografi-

1 So ergab sich beispielsweise bei einer demografischen Analyse in einem mittelständischen Unternehmen durch einen der Autoren, dass die Annahme von 2\% Wachstum des Unternehmens kumuliert über sieben Jahre erhebliche höhere Auswirkungen auf die Personalplanung hatte, als binnendemografische Entwicklungen im selben Zeitraum.

2 www.arbid.de 
schen Wandels auf die Unternehmen skizziert werden. Dabei handelt es sich um die Absatzperspektive, die personalwirtschaftliche Perspektive sowie übergreifende gesellschaftliche Aspekte.

\subsection{Absatzperspektive}

In der Absatzperspektive wird danach gefragt, welche Risiken und Chancen sich für die Nachfrage nach bestehenden, aber auch innovativen Produkten und Dienstleistungen ergeben. In erster Linie betrifft dies alle Unternehmen, die nah am Endverbraucher agieren (zum Beispiel Konsumgüterindustrie, Handel, Tourismus). In zweiter Linie deren Zulieferer und Dienstleister ${ }^{3}$. In einer erweiterten Perspektive aber auch Unternehmen, die Lösungen zur Produktivitätssteigerung herstellen ${ }^{4}$. In bestimmten personalintensiven Dienstleistungsbereichen wie Kranken-und Altenpflege oder Einzelhandel kommt es darüber hinaus zu dem Effekt, dass demografische Veränderungen sozusagen auf „beide Seiten des Tresens“ durchschlagen ${ }^{5}$.

\subsection{Personalwirtschaftliche Perspektive}

Die Deckung des Fachkräftebedarfs wird zu einem der zentralen Aspekte in der personalwirtschaftlichen Perspektive werden. Eine schrumpfende Bevölkerung mit weniger jungen Menschen führt zu einem Rückgang der Schulabgänger und Berufsanfänger. Momentan wird diese Tatsache durch die öffentliche Diskussion um fehlende Ausbildungsplätze noch verdeckt, doch bereits in wenigen Jahren wird sich dieser Trend in sein Gegenteil verkehren. Neben der quantitativen Betrachtung muss ebenfalls die Frage aufgeworfen werden, inwieweit die Qualifikation der potentiellen Berufsanfänger den Anforderungen der Unternehmen entsprechen wird. Die verschiedenen Bildungsstandserhebungen der letzten Jahre zeichnen hier kein allzu optimistisches Bild. Gleichzeitig haben wir es jedoch in weiten Teilen der Wirtschaft mit einem anhaltenden „Upskilling-Prozess“ zu tun, der stetig höhere Qualifikationen in einem weltweiten Innovationswettbewerb fordert ${ }^{6}$. Die Lücke zwischen Bedarf und Angebot droht derzeit größer statt kleiner zu werden. Insbesondere kleine und mittlere Unternehmen mit traditionell regionaler Rekrutierung, einfachen Rekrutierungsinstrumenten und ohne ausgeprägtes Personalmarketing drohen dann beim „Wettbewerb um die Köpfe“ ins Hintertreffen zu geraten.

Die Erhaltung und Steigerung der Produktivität alternder Belegschaften bildet den zweiten Schwerpunkt. Während in der volkswirtschaftlichen Perspektive die Leistung an erzeugten Gütern und Diensten pro Kopf mit der Zahl der Erwerbstätigen multipliziert wird, steht bei der betriebswirtschaftlichen Perspektive die individuelle Produktivität pro Zeiteinheit im Mittelpunkt. Die Kernfrage lautet: Gibt es einen Zusammenhang zwischen Lebensalter und Leistung? Und sind damit im statistischen Mittel ältere Belegschaften per se leistungsschwächer? Für den Bereich der krankheitsbedingten Leistungsausfälle liegt recht umfangreiches Datenmaterial vor. Hier kann gesagt werden, dass zwar Ausfälle - und damit Produktivitätsminderungen - mit dem Lebensalter zunehmen, dies aber vor allem für chronische Erkrankungen zutrifft und zudem deutlich nach Berufsgruppen und Tätigkeiten differenziert.

Für den Bereich der „gesunden Älteren“ kann jedoch derzeit keine abschließende Feststellung getroffen werden. Hinweise finden sich sowohl für die Idee einer kompensatorischen Kompetenzentwicklung, bei der nachlassende Fähigkeiten durch andere ersetzt werden können, als auch für die Annahme eines ,umgekehrt-U-förmigen“ Verlaufes, nach dem trotz allem mit einem Nachlassen der Leistung zu rechnen ist. Empirisch ist diese Frage derzeitig nicht zu beantworten, denn die Vielfalt an Arbeitsinhalten und Arbeitsbedingungen, Leistungsarten und Messproblemen, individuellen Unterschieden, Einflüssen von Sichtweisen, Fragestellungen und Untersuchungsdesigns, etc. ist dafür zu groß $\aleph^{7}$.

Unstrittig ist jedoch, dass die individuellen Unterschiede zwischen Menschen mit dem Lebensalter zunehmen. Ob dies nun biologische Gründe hat oder mit (berufs-)biografischen Unterschieden zu erklä-

3 Man denke hier zum Beispiel an die Bemühungen der Werbewirtschaft, wirksame Werbebotschaften für ältere Kunden zu kreieren.

4 Vgl.: Remarks by Chairman Alan Greenspan. Federal Reserve Bank of Kansas City’s symposium on "Global Demographic Change: Economic Impacts and Policy Challenges”. Jackson Hole, Wyoming, August 26-28, 2004

5 So wird in einem Beitrag in einem Wirtschaftsmagazin Hans-Joachim Körber, der Vorstandsvorsitzende der Metro zitiert: „Ältere Menschen wollen gerne von Gleichaltrigen bedient werden, sagt Körber, der bei der Metro den Vorruhestand abschaffte.“ (CAPITAL, Nr. 8/2005, S. 57).

6 Siehe aktuell zum Begriff des Upskilling und der dahinter liegenden Entwicklung am Beispiel der Metall- und Elektro-Industrie: Institut der Deutschen Wirtschaft: „Die M+E-Industrie in Deutschland“. Köln, Februar 2005, S. $68 \mathrm{ff}$.

7 Vgl. Börsch-Supan, A. u.a.: „Altern und Produktivität: Zum Stand der Forschung“. MEA Discussion Paper 732005, Mannheim, Mai 2005. 
ren ist oder ob gar eine Mischung beider Faktoren vorliegt, ist dann zweitrangig, wenn aktuell in einem Unternehmen faktische Produktivitätseinbußen befürchtet werden oder bereits festzustellen sind. Hier haben Intervention, die Verbesserung des gegenwärtigen Zustandes sowie Prävention Vorrang.

Wie wird sich schließlich die Unternehmenskultur verändern (müssen), in Anpassung an die skizzierten Entwicklungen und Probleme? Es ist klar, dass Kulturen, in denen Ältere als weniger produktiv und kreativ betrachtet werden und dies auch gezeigt wird (z.B. durch Ausschluss von Weiterbildungsmaßnahmen), kaum langfristig zukunftsfähig sein werden. Nichtsdestoweniger werden sie dort, wo sie anzutreffen sind, nicht über Nacht zu überwinden sein. Das Verschwinden von gesetzlicher Grundlage und betrieblicher Praxis der Frühverrentung trägt ebenfalls zu erheblichen kulturellen Brüchen in den Unternehmen bei. Ganze Alterskohorten im Bereich $50+$ müssen aktuell und zukünftig wesentlich länger im Betrieb verbleiben. Schließlich sind auch zunehmende Verteilungskonflikte zwischen verschiedenen Generationen im Unternehmen nicht auszuschließen $^{8}$.

\subsection{Gesellschaftliche Rahmenbedingungen}

Neben den beschriebenen direkten Wirkungen auf die Absatzseite und die personalwirtschaftliche Seite gibt es bedeutsame indirekte Wirkungen auf Unternehmen durch gesellschaftliche Entwicklungen. So würden sich Alter und Zusammensetzung des Personals zwangsläufig ändern, wenn durch gesetzliche und andere Maßnahmen die Beschäftigungsquoten bestimmter Gruppen am Arbeitsmarkt (z.B. Frauen, Migranten, Ältere) signifikant erhöht würden. Beispielsweise ist auf mittlere Sicht eine Anhebung des gesetzlichen Renteneintrittsalters zumindest nicht auszuschließen. Eine weitere Maßnahme stellt der zur Zeit kontrovers diskutierte Gesetzesentwurf gegen zivil- und arbeitsrechtliche Diskriminierung dar, der Diskriminierung aufgrund des Alters als Tatbestand einschließt. Aber auch Medien, die öffentliche Meinung oder Interessengruppen Älterer sind in der Lage, die Rahmenbedingungen der Unternehmen zumindest mittelbar zu beeinflussen.

Schließlich wird sich auch die Frage nach der Innovationsfähigkeit alternder Gesellschaften mit zunehmender Dringlichkeit stellen. Müssen wir mit mehr Alten und weniger Jungen auch mit einem Nachlassen der Kreativität, des Ideenreichtums, der schöpferischen Kraft rechnen? Und wenn ja, was würde das konkret bedeuten für so unterschiedliche wirtschaftliche Akteure wie Forschungs- und Entwicklungsabteilungen, Werbeagenturen, Gießereien oder den Einzelhandel? Dass diese Frage derzeit nicht hinreichend geklärt ist, kann man aktuelle einer eher pessimistischen Aussage von Paul Baltes entnehmen: „Kann man aus den ... Forschungsbefunden etwas über das ... Denk- und Innovationspotenzial von Gesellschaften mit unterschiedlichen $\mathrm{Al}$ tersstrukturen ableiten?... Prognose: Die höhere Altersstruktur in Deutschland, gepaart mit einer größeren Zahl von Alten als Jungen, ist ein Risikofaktor." “9

Auch hier müssen wir also derzeit gestehen: wir wissen zu wenig, denn noch nie vor uns ist eine technisch hochgerüstete, auf Innovationen angewiesene Volkswirtschaft einer derartigen demografischen Veränderung unterzogen worden.

\section{Die Initiative der Sozialpartner in Nordrhein-Westfalen}

\subsection{Ziele und Partner der Initiative}

Aus dem Gesagten ist klar geworden, dass die Bewältigung des demografischen Wandels in den entwickelten Industriestaaten, in Deutschland und in Nordrhein-Westfalen zu den zentralen Herausforderungen gehört, auf die eine Strategie zur nachhaltigen Sicherung von Arbeitsplätzen und Wohlstand Antworten geben muss. Um zukunfts- und wettbewerbsfähig zu bleiben, müssen die Betriebe bereits heute eine entsprechende Arbeits- und Personalpolitik entwickeln. Sich auf den demografischen Wandel einzustellen, ist nicht nur eine sozialpolitische, sondern vor allem auch eine wettbewerbspolitische Frage. Die Sozialpartner wollen daher mit ihrer Initiative und zusammen mit weiteren Partnern dazu beitragen, dass:

- die Unternehmen Chancen und Risiken des demografischen Wandels erkennen,

- $\quad$ kompetente Ansprechpartner für Unternehmen in Verbänden, Gewerkschaften sowie Bildungsund Beratungseinrichtungen bereit stehen und

- Beispiele guter Praxis, erfolgreiche Strategien und bewährte Methoden verbreitet werden.

Bislang konnten bereits wichtige Schritte beim Aufbau einer tragfähigen Initiative getan werden. Dazu zählen vor allem:

- die gemeinsame Verständigung der Partner hinsichtlich der Ziele und Aufgaben sowie die Etablierung von Kommunikations- und Kooperationsstrukturen nach innen und nach außen,

8 Eine Idee davon liefert Jörg Tremmel: „Die fetten Jahre sind vorbei...für die Jüngeren“. DIE ZEIT, Nr. 14, 2005.

9 DIE ZEIT, Nr. 21, 19.05.2005. 
- die Gestaltung von entsprechenden Kommunikationsmedien,

- die Erarbeitung von Materialien zur Unterstützung von Akteuren ${ }^{10}$,

- der Aufbau eines arbid Kompetenzpools aus Projekten und Dienstleistern in NRW,

- Information und Schulung von Unternehmensvertretern, Betriebsräten und Intermediären durch Publikationen, Vorträge und Seminare

Im Rahmen des Projektes „Kompetenzentwicklung in Betrieben mit alternden Belegschaften " ${ }^{11}$ konnten Unternehmen aus NRW für eine aktive Mitarbeit gewonnen werden. Neben der Erprobung von Methoden durch die Unternehmen erwiesen sich vor allem die rege Beteiligung der Unternehmen in den überbetrieblichen Aktivitäten als sehr förderlich für die Entwicklung der Initiative.

Das zunehmende Interesse von betrieblichen Akteuren, Vertretern der Sozialpartner auf regionaler Ebene, Dienstleistern und Regionen an den Aktivitäten und Angeboten von arbid zeigt, dass die Initiative einem realen und stetig wachsenden Bedarf gegenüber steht.

\section{$4 \quad$ Aktuelle Ansatzpunkte der Initiative}

\subsection{Strategische Ansätze in Unternehmen}

Strategische personalwirtschaftliche Ansätze zur Bewältigung des demografischen Wandels sind derzeit vorwiegend in Großunternehmen anzutreffen. Solche Unternehmen sind daran interessiert zu erfahren, welche spezifischen demografisch bedingten Risiken für das Unternehmen bestehen und ob auch zukünftig die Leistungserstellung aus personalwirtschaftlicher Perspektive als gesichert angesehen werden kann. Neben diesem Blick nach innen wird dabei auch durchaus der Blick nach außen gerichtet und eine demografische Risiko- und Potentialanalyse wird in der Kommunikation mit Banken und Investoren als Beleg der Nachhaltigkeit der unternehmerischen und personalpolitischen Strategien verwendet.

Entsprechende Werkzeuge und Erfahrungen liegen heute bereits vor und können von interessierten Unternehmen übernommen und gegebenenfalls auf ihre speziellen Bedürfnisse hin angepasst werden ${ }^{12}$.
Es wird daher in dieser Hinsicht für arbid verstärkt darum gehen, durch eine gezielte Öffentlichkeitsarbeit weitere Unternehmen zu sensibilisieren. Durch zusätzliche Informationsangebote und „Einsteigerinstrumente" kann die Wahrnehmungs- und Anwendungsschwelle zusätzlich gesenkt werden.

Aus der Sicht der Mitarbeiter sind Beschäftigungsfähigkeit und -sicherung zentrale Anliegen. Dies betrifft zum einen den Erhalt der eigenen Gesundheit, bei Wegfall von Frühverrentungsperspektiven und einer eventuell ja verlängerten Lebensarbeitszeit. Insbesondere bei belastenden Arbeitsbedingungen (z.B. durch schwere körperliche Arbeit, ungünstige Körperhaltungen, Umgebungsbedingungen) besteht hier Handlungsbedarf, zumal auch Möglichkeiten der innerbetrieblichen Umsetzung auf weniger belastende Arbeitsplätze tendenziell deutlich geringer werden. Hier sind alternative Strategien im Zusammenspiel betrieblicher und außerbetrieblicher Akteure erforderlich.

Zum anderen steht auch bei Erhalt der Gesundheit die Frage der dauerhaften Beschäftigungsfähigkeit im Raum. Da eine bruchlose Beschäftigung in einem Betrieb oder an einem Arbeitsplatz von der Regel zunehmend zur Ausnahme wird, muss der Mitarbeiter über arbeitsmarktfähige Kompetenzen verfügen, die einen entsprechenden Wechsel möglich machen. Erwerb und Erhalt, Messung und Dokumentation solcher Kompetenzen in effizienter und finanzierbarer Weise sind daher ein wichtiges Anliegen.

\subsection{Beiträge zur Entwicklung des Wissens- und Methodenstandes}

Die Initiative sammelt und analysiert Informationen und Forschungsergebnisse zum demografischen Wandel und bereitet diese für Unternehmen, Partner und Dritte auf. In Konferenzen und Workshops trägt arbid die Sichtweise von Unternehmen und Beschäftigten bei. arbid will damit insbesondere zu einer Versachlichung und Lösungsorientierung der komplexen öffentlichen Diskussion beitragen. Im Rahmen der „Benchmarkingstudie demografischer Wandel und Produktivitätsentwicklung in der Metall-und Elektroindustrie in NRW“ 11 befasst sich die GOM in Kooperation mit dem Institut für angewandte Arbeitswissenschaft in Köln (IfaA) mit der Frage der

10 Technologieberatungsstelle beim DGB NRW e.V. (Hrsg.): „Alternde Belegschaften im demografischen Wandel. Ein Thema für Interessenvertretungen. Reihe Arbeit, Gesundheit, Umwelt, Technik, Heft 64 Oberhausen, 2005. Institut für angewandte Arbeitswissenschaft (Hrsg.): „Demografische Analyse und Strategieentwicklung im Unternehmen.“ Köln, 2005

11 Gefördert aus Mitteln des Landes NRW und der EU

12 Vgl. Mühlbradt \& Schawilye: „Analyse personalwirtschaftlicher Risiken und Potenziale“; in: Institut für angewandte Arbeitswissenschaft (Hrg.): Demografische Analyse und Strategieentwicklung im Unternehmen. Köln, 2005 
Produktivität alternder Belegschaften. Vor diesem Hintergrund fand auf Einladung der Projektpartner im Juni dieses Jahres unter anderem der Workshop „Produktivität im demografischen Wandel: Konzepte, Erkenntnisse und Impulse “ statt, zu dem Experten aus den Bereichen Arbeitswissenschaft, Volkswirtschaft, Arbeitsmedizin sowie Vertreter der Politik und der Sozialpartner teilnahmen. Dabei wurden, disziplinenübergreifend, Perspektiven, Herangehensweisen und aktuelle Erkenntnisse zum Problemfeld vorgestellt und diskutiert.

Die TBS verfolgt im Rahmen des Projektes „Beschäftigungsfähigkeit in Betrieben“ das Ziel, das Leitkonstrukt der Beschäftigungsfähigkeit in Form von Checklisten für Unternehmen und Beschäftigte in ein praktikables Instrument umzusetzen ${ }^{13}$. Damit wird ein Beitrag geleistet zur Implementierung von Ansätzen zum Erhalt der Beschäftigungsfähigkeit alternder Belegschaft, der über die reine Arbeitsfähigkeit hinausgeht ${ }^{14}$.

\subsection{Transfer: Demografischer Wandel im Unternehmen als Beratungs- und Bildungsfeld}

Betrachtet man schließlich die geografische Ausdehnung des Landes Nordrhein-Westfalen, die Vielzahl verschiedener Regionen und die enorme Anzahl an Unternehmen unterschiedlicher Größe und Branchenzugehörigkeit, so braucht arbid kompetente Mitstreiter unter den Anbietern von Bildungs- und Beratungsdienstleistungen im Land. Dies umfasst beispielsweise folgende demografische Handlungsfelder:

- Schulungs- und Beratungsangebote sowie Trainings im Bereich der (über)betrieblichen Gesundheitsprävention und der Gesundheitsförderung sowie der ergonomischen Analyse und Gestaltung von Arbeitsplätzen und -bedingungen

- Angebote im Bereich der beruflichen Bildung, insbesondere der Weiterbildung von Beschäftigten, der Qualifizierung von älteren und lernungewohnten Mitarbeitern, der Entwicklung und Umsetzung von innovativen Ansätzen der Kompetenzentwicklung in der Arbeit im betrieblichen und überbetrieblichen Kontext

- Angebote zum Kulturmanagement im Sinne des Multigenerationenunternehmens, der age awareness bei Führungskräften, Personalfachleuten und Mitarbeitern und des diversity managements arbid informiert die Akteure über Themen und damit verbundene Chancen für die Leistungsvermarktung und fördert den Informations- und Erfahrungsaustausch sowie den Aufbau von Kooperationen zwischen den Akteuren.

\section{Dr. Thomas Mühlbradt}

GOM Gesellschaft für Organisationsentwicklung und Mediengestaltung $\mathrm{mbH}$

Buchkremerstraße 6

D-52062 Aachen

t.muehlbradt@gom.de

\section{Dr. Jürgen Grumbach}

Technologieberatungsstelle beim DGB NRW e.V. Lothringer Str. 62

D-46045 Oberhausen

tbs-hauptstelle@tbs-nrw.de

3 Projekt „Beschäftigungsfähigkeit in Betrieben“, gefördert mit Mitteln des Landes NRW und der EU www.bib.de.

14 Vgl. dazu Naegele, G.: „Nachhaltige Arbeits- und Erwerbsfähigkeit für ältere Arbeitnehmer“; in: WSI Mitteilungen 4/2005, 214-219. 